\title{
4
}

\section{MODIFIED SHAPE ANNEALING FOR OPTIMALLY-DIRECTED GENERATION: INITIAL RESULTS}

\author{
KEN N. BROWN ${ }^{\S}$ AND JON CAGAN \\ Carnegie Mellon University, USA
}

\begin{abstract}
Formal generative design systems may describe large and complex spaces, making good designs hard to find. The problem of directing generation towards good solutions is addressed by considering the shape annealing algorithm. The algorithm is modified in an attempt to make optimization over the final generation sequence more uniform. Seven variations of the algorithm are investigated, and their performance compared. The results suggest that an enhanced backtracking ability may significantly improve the performance of the algorithm.
\end{abstract}

\section{Introduction}

Formal generative systems promise a number of advantages for a science of design. They provide an executable specification of a design space, supporting the generation of designs within the space. They offer, in principle, an analyzable definition of the design space, allowing candidate designs to be recognized as elements of the space. They provide (again in principle) a means to analyze properties of the spaces themselves. They offer a formal basis for reasoning about designs in progress, via their semantics. In order to meet these promises, a number of questions have to be answered.

Although formal systems can be concise and elegant descriptions of design spaces, the spaces themselves can be vast and the relationship between the description and the designs its specifies unclear. The result of applying sequences of individual rules is not obvious. How is a designer to know what designs exist in the space, and which regions of the space contain what types of designs? How is a designer to find good designs within the space? How should the syntax of a system be interpreted, and in particular, how should partial, incomplete designs in the space be interpreted? Intelligent generation within a space would seem to require either a knowledge of the space's overall structure, or some method of judging intermediate states and making

\footnotetext{
${ }^{\S}$ Now at University of Aberdeen, Scotland.
} 
decisions about which directions to follow. More complete discussions of these issues are given by Carlson (1994b) and Cagan (1994b).

One technique that has been proposed for searching large design spaces is shape annealing (Cagan and Mitchell, 1993), a search method directed towards optimal solutions, based on the stochastic optimization technique of simulated annealing (Kirkpatrick et al., 1983). Shape annealing is useful in cases where the intermediate assessments are such that simply applying the locally best operation at each stage does not necessarily lead to the best final designs, as its stochastic nature allows it to backtrack out of local optima. Shape annealing produces good solutions efficiently; however, it is not guaranteed to find the optimum, and seems to find it only rarely. In this paper, we consider why this should be so, and suggest some modifications to the basic technique in order to make reaching the optimum more likely.

\section{Background}

The use of grammars (formal generative systems) for the design of complex structures was popularized by the development of the shape grammar formalism (Stiny, 1980). In this formalism, rewrite-rules are recursively applied to two-dimensional shapes to produce languages of two-dimensional shape designs. Parametric shape grammars can also be defined, in which rule schemas with variable parameters are specified, and rule application proceeds with an instantiated version of one of the rule schemas. A number of shape grammars have been presented in the literature, including a grammar of Palladian villas (Stiny and Mitchell, 1978) and a grammar of Queen Anne houses (Flemming, 1986). A general definition of the algebra of shapes is given by Stiny (1991). Grammars were originally used as a means of analyzing and understanding existing corpuses of designs, by showing that the designs conform to a regular structure.

Viewed in the abstract, the whole design process can be considered to be generation. Stiny and March (1981) proposed design machines, while Fitzhorn (1989) has proposed a formal computational theory of design. In both of these papers, the design process is modeled as the interaction of constraints and the design context with the grammar rules used to generate the designs. Brown et al. (1993b) discuss some possible roles for grammatical methods in engineering design, in the context of a transformational model of design (McMahon et al., 1993). Recently, there has been some effort in building grammatically-based design tools, allowing designers to explore design spaces interactively (Carlson, 1994a; Heisserman and Woodbury, 1994).

The use of grammars as design tools raises the question of how designs should be interpreted. The conventional view of the semantics of a design representation provides a mapping from the elements of the representation 
to real world objects. We can also look at performance criteria, or objectives, as the semantics of a design, either in terms of rigorous mathematical notions of performance, or the more fluid and subjective assessments of human users. We can use the constructive nature of grammatical tools to construct these semantics in tandem with the syntactic generation. Stiny (1981) proposed the generation of design descriptions by associating rules operating on descriptions with the grammar rules which operate on the shapes. Brown et al. (1994a, 1994b) have applied variants of this formalism to the interpretation of engineering artifacts in terms of feature descriptions and manufacturing plans. For string and graph based formalisms, attribute grammars (Knuth, 1968) have been used, in which attributes representing additional information augment symbols, and attribute rules define the computation of the attribute values. Variations on this theme are proposed by Penjam (1990), representing the resistance of electrical circuits, Rinderle (1991), representing forces and weights of boom designs, and Brown et al. (1992), representing stress concentrations on loaded shafts. However, these applications are oriented towards providing a final interpretation or assessment of a design. When used in design tools, the assumption is that the partial interpretation constructed as a design is in progress can be used to direct generation towards a desired end result. This is not necessarily the case, and depends on the particular semantic functions being used. The problem remains for each particular design space and design task of determining objective functions with which variations in the intermediate assessments after individual rule applications accurately reflect the variations in the assessment of the final designs resulting from those rules.

The question then arises of how best to guide search or exploration through these design spaces, which are generally too large for exhaustive search to be practical. For exploration, random sampling of the space may suffice, although as Carlson (1994b) points out, for a truly uniform sample of the space, the frequency with which particular rules are applied in particular situations should depend on the size of the sub-space below the states that result. For search in relatively small spaces, standard artificial intelligence search algorithms may be applied. For larger spaces, Cagan and Mitchell (1993) proposed shape annealing as a means of directing search towards optimal solutions. Shape annealing attempts moves more or less at random which are accepted according to a steadily more discriminating acceptance criterion. Thus, a search in a design space consists of successive rule applications and retractions which are initially randomly selected and accepted almost regardless of their effect on the objective function. As time progresses, moves which degrade the objective function are accepted with decreasing probability. Eventually, the algorithm converges to a good local optimum. Because shape annealing is stochastic, it is able to recover from 
early, poor local optima. Thus, intermediate objective functions do not need to be strictly accurate, as moves which initially appear to take us closest to our goal but later turn out to have been non-optimal are not fatal to the algorithm. However, the choice of intermediate objective function is still crucial to the algorithm's success. Shape annealing has been applied to component layout (Szykman and Cagan, 1994), the generation of truss topologies (Reddy and Cagan, 1994), and geometric knapsack problems (Cagan 1994a).

As stated earlier, although shape annealing is an optimally-directed search technique, and produces good solutions, the final designs are rarely the global optimum, and in many cases are far from it. In the following section, we discuss the algorithm in more detail, and consider why this might be the case.

\section{Shape Annealing}

Let $\mathrm{G}=(\mathrm{S}, \mathrm{L}, \mathrm{R}, \mathrm{I})$ be a shape grammar, consisting of a set of shapes, $\mathrm{S}$, a set of labels, $L$, a set of shape rules, $R$, and an initial labeled shape I. Let $f:(S, L)^{+}$ $\rightarrow \mathbf{R}$ be the objective function, mapping labeled shapes to real numbers. Let $\mathrm{T}$ be a real-valued variable, called the temperature.

A state $s_{i}$ is a labeled shape obtainable by recursively applying a sequence of $n$ rules from $R$ to $I(n \geq 0)$. Let $f\left(s_{i}\right)=C_{i}$ A shape annealing move is then the application of a grammar rule $r_{j}$ to $s_{i}$ or the retraction of the last rule applied to obtain $s_{i}$, to obtain the labeled shape $s_{i+1}$. Let $f\left(s_{i+1}\right)$ $=C_{i+1}$. If $C_{i+1}<C_{i}$, then $s_{i+1}$ is accepted as the next state. If $C_{i+1}>C_{i}$, then $s_{i+1}$ is accepted as the next state with a probability defined by

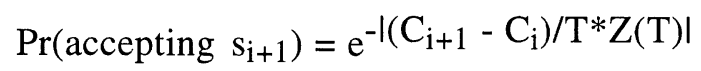

where $\mathrm{Z}(\mathrm{T})$ is a normalization factor. $\mathrm{T}$ decreases with time.

Although it can be proven that simulated annealing under certain conditions relating to the parameters of the algorithm will always find the global optimum, the conditions are unfeasibly restrictive for real applications. At least the same restrictions apply to shape annealing. However, it appears that when tested on the same problem, shape annealing can produce inferior solutions to standard simulated annealing (Szykman and Cagan, 1994). There are two main reasons for this. Firstly, as stated above, the choice of objective function for intermediate designs is significant. It appears to be harder to relate the early stages of a design to the final design and make decisions on that basis than it does to represent violations of constraints in fully instantiated designs and minimize those violations. Secondly, there is a subtle difference in the implications of accepting a move in the two algorithms. This point is discussed below. 
In the original shape annealing application of Cagan and Mitchell (1993), a design is produced by a sequence of moves, each one of which constrains the subspace that is subsequently reachable. Each step in a generation can thus be said to contribute to the final cost of the design by imposing constraints on the costs that can be reached. After accepting a particular move, in order to generate a design in the subspace that is outside the resulting subspace, the shape annealing algorithm has to backtrack over the moves responsible. The length of the move sequence generally grows with time. However, the acceptance criterion becomes more discriminating with time as the temperature drops. Thus decisions on whether rule applications and retractions are accepted are not uniform over the length of the sequence, and optimization is concentrated towards the end. If we had complete knowledge of the design space and the objective function (i.e. we could characterize completely the relationships between the states in the space and the objective functions of the final designs below them) we would see that the optimality of the individual moves increases with the length of the sequence. This phenomenon was referred to implicitly in the original paper by Cagan and Mitchell (1993), in which they discussed the way in which the results are crucially dependent on the early choice of moves. In other words, if we are fortunate in our initial choices, we end up with a solution close to the optimum; if we are unfortunate, then no amount of optimization at the end of the sequence will produce the optimum. (This is not to say that the early moves in the sequence are randomly chosen, as the algorithm frequently backtracks over those moves and tries alternatives. It is simply that when the algorithm is concentrating on the early stages, the acceptance criteria is loose, and as the criteria becomes more stringent and the sequence lengthens, the algorithm is less able to backtrack long distances). In addition, the algorithm has no memory of the states it has visited, and once it has backtracked out of a superior path, it has no knowledge of the previous objective functions it had discovered and may subsequently settle on poorer states. Finally, it should be noted that shape annealing in itself is not the cause of the above behavior, but its application to generative systems in which each move narrows the space of reachable designs. These arguments do not apply to systems in which any state can be reached from almost any other state by forward rule applications - for example, Reddy and Cagan's (1994) truss topology grammar. In this paper, we restrict our comments to those systems in which a forward move does limit our space of designs.

These comments suggest a number of possibilities for improving the algorithm. One is to ensure that the objective functions at the early stages better reflect the utility of the sub-spaces that the moves create, either by finding a better objective function, or by changing the grammar so that the 
initial moves create better partitions of the whole space. A second is to implement some form of memory, so that previous objective function evaluations are incorporated in some way into the algorithm. A third to is change the move set available to the algorithm to try to compensate for the non-uniform optimization. Cagan and Kotovsky (1994) have investigated the propagation of objective function evaluations from states to neighboring states, effectively combining the memory approach with the improved objective function approach. Schmidt and Cagan (1994) have considered recursive annealing, in which design progresses through different levels of abstraction, annealing at each stage, and thus providing better estimates of subsequent costs when deciding upon moves at higher levels. For the remainder of this paper, we will consider the third option, and investigate alternative move sets.

\section{Experiments}

In the experiments described below, we had two main aims: to allow the acceptance criterion to use information about the costs of subsequent states in the algorithm, and to increase the likelihood of the algorithm backtracking out of deep local optima. We compared seven different backtracking strategies, sketched in Figure 1, and described below. In what follows, we will refer to the current state as $s_{\text {old }}$, and the state the algorithm is attempting to move to as snew.

$\mathrm{s}_{\text {old }}$ is a state obtained by applying rules $\left(\mathrm{r}_{i_{1}}, \mathrm{r}_{i_{2}}, \ldots, \mathrm{r}_{\mathrm{i}_{n}}\right)$ to the initial shape. Let $\mathrm{C}_{\text {old }}$ be the old evaluated cost and $\mathrm{C}_{\text {new }}$ be the new evaluated cost used in the probability calculation.

A. $s_{\text {new }}$ is the state obtained by applying a rule $r_{i_{n+1}}$ to $s_{o l d}$, or by retracting $\mathrm{r}_{\mathrm{i}_{\mathrm{n}}} \cdot \mathrm{C}_{\text {old }}=\mathrm{f}\left(\mathrm{s}_{\text {old }}\right), \mathrm{C}_{\text {new }}=\mathrm{f}\left(\mathrm{s}_{\text {new }}\right)$.

B. $s_{\text {new }}$ is the state obtained by applying a rule $r_{i n+1}$ to $s_{\text {old }}$, or by retracting rules $r_{i_{n}}$ back to $r_{i j}$, for $1 \leq j \leq n . C_{\text {old }}=f\left(s_{\text {old }}\right), C_{\text {new }}=f\left(s_{\text {new }}\right)$.

C. $s_{n e w}$ is the state obtained by applying a rule $r_{i_{n+1}}$ to $s_{\text {old }}$, or by retracting rules $r_{i_{n}}$ back to $r_{i j}$ to get state $s_{j}$, followed by applying a rule $r_{i j}{ }^{\prime}$ to $s_{j} \cdot C_{\text {old }}=$ $f\left(r_{i j}\left(s_{j}\right)\right), C_{n e w}=f\left(s_{n e w}\right)$.

D. $s_{n e w}$ is the state obtained by applying a rule $r_{i_{n+1}}$ to $s_{\text {old }}$, or by retracting rules $r_{i_{n}}$ back to $r_{i_{j}}$ to get state $s_{j}$, followed by applying a rule $r_{i_{j}}{ }^{\prime}$ to $s_{j}$. $C_{o l d}=$ $\mathrm{f}\left(\mathrm{s}_{\text {old }}\right), \mathrm{C}_{\text {new }}=\mathrm{f}\left(\mathrm{s}_{\text {new }}\right)$. 


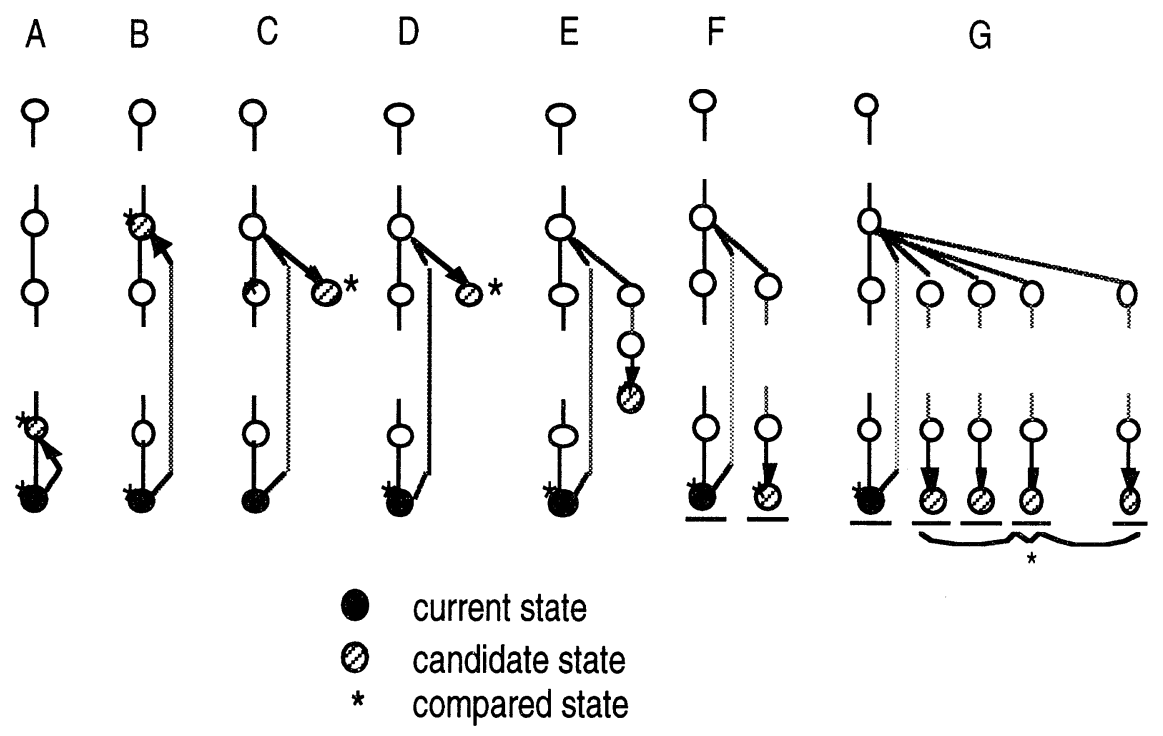

Figure 1. Alternative backtracking moves.

E. $s_{n e w}$ is the state obtained by applying a rule $r_{i_{n+1}}$ to $s_{\text {old }}$, or by retracting rules $r_{i_{n}}$ back to $r_{i_{j}}$ to get state $s_{j}$, followed by applying rules $r_{i_{j}}{ }^{\prime}, \ldots, r_{i_{m}}{ }^{\prime}$, such that $m-j=\min (n-j, x)$ for some fixed $x . C_{o l d}=f\left(s_{o l d}\right), C_{n e w}=f\left(s_{n e w}\right)$.

F. $s_{\text {old }}$ is a finished design. $s_{\text {new }}$ is the finished design obtained by retracting rules $r_{i_{n}}$ back to $r_{i j}$, for $1 \leq j \leq n$, followed by applying rules $r_{i_{j}}{ }^{\prime}, \ldots, r_{i_{m}} \cdot C_{o l d}$ $=f\left(s_{\text {old }}\right), C_{\text {new }}=f\left(s_{\text {new }}\right)$.

G. $\mathrm{s}_{\text {old }}$ is a finished design. $\mathrm{s}_{\text {new }}$ for all $\mathrm{k}$ such that $1 \leq \mathrm{k} \leq \mathrm{p}$, for some fixed $p$, is the finished design obtained by retracting rules $r_{i_{n}}$ back to $r_{i j}$, for $1 \leq j$

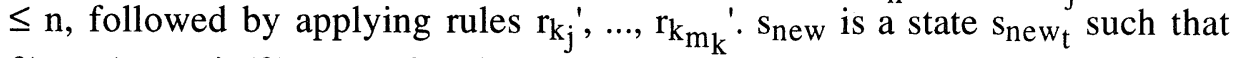
$\mathrm{f}\left(\mathrm{s}_{\text {new }_{\mathrm{t}}}\right)=\min \left(\mathrm{f}\left(\mathrm{s}_{\text {new }_{\mathrm{k}}}\right)\right)$ for all $\mathrm{k}: 1 \leq \mathrm{k} \leq \mathrm{p} \cdot \mathrm{C}_{\text {old }}=\mathrm{f}\left(\mathrm{s}_{\text {old }}\right), \mathrm{C}_{\text {new }}=\mathrm{f}\left(\mathrm{s}_{\text {new }}\right)$.

Move set $\mathrm{A}$ is the original shape annealing algorithm. $\mathrm{B}$ is intended to make it easier to back out of local optimum, by allowing the retraction of an arbitrary number of the applied rules. Although the probability of acceptance will be lower than for any single backtrack step, it should be higher than the accumulated probability of accepting the equivalent sequence of single backtracking steps. For C and D, it was noted that backtracking involves the loss of useful information. Suppose the transition from $s_{i}$ to $s_{i+1}$ was a good transition, but that we backtrack to state $s_{i}$. The algorithm will now accept any move that results in a better state than $s_{i}$, even though it may be much worse than $s_{i+1}$. We need some way of balancing the 
ability to backtrack out of local optima with the retention of information from previous moves. Thus for $\mathrm{C}$, a backtracking move consists of the retraction of a number of rules to state $s_{i}$, followed by a forward rule application to give a new state $s_{i+1}$ '. Comparison of costs for the acceptance criterion is then between $s_{i+1}$ and $s_{i+1}$. D is the same as $C$, except that comparison is between $s_{n}$ and $s_{i+1}$ '. E extends this "look forward" idea by applying a number of forward rule applications (up to a limit) before comparison. $\mathrm{F}$ is motivated by an attempt to use as good an estimate of the final objective functions as possible. The best possible estimate is the objective function for finished designs - therefore, in F, comparisons are only made between completed designs. This algorithm first randomly applies rules until a completed design is obtained; an annealing move then consists of a backtrack to state $s_{i}$, followed by a new random completion from $s_{i}$ to $s_{m}{ }^{\prime}$. The objective function values for $s_{n}$ and $s_{m}$ ' are then compared as normal. Finally, $\mathrm{G}$ is based on F, except that after a backtrack, a fixed sample of completions are generated, and the best completion is used for comparison.

In order to test the various moves sets, we used a simple generative system as a test problem. The problem was devised to have a seemingly obvious optimum move at every stage, but with a small number of local optima. The system generates descending lists of real numbers from 100 to 0 . Given a partial list, a move consists of simply selecting the next element. Generally, a move involves subtracting a number between 1 and 5 (in integer multiples of 0.01 ) from the current last element, $X_{i}$, to obtain the next element $X_{i+1}$, such that $X_{i+1} \geq 0$. However, there are a few exceptions. If $X_{i}$ is between 61 and 62 , then $X_{i+1}=55$. If $X_{i}$ is between 60 and 61 , the $X_{i+1}=59.99$. If $X_{i}=$ 59.99, then $X_{i+1}=59.98$. Corresponding exceptions apply to values in the range 29.99 to 32 . A more formal description of the system is given in the appendix.

Our design task will be to generate short lists, and thus the objective function for a completed list is simply its length minus 1 . The optimum cost is 20 , and there are many lists with that cost, for example,

$$
<1009590858075706561.5555045403531 .22520151050>\text {. }
$$

Note that without knowledge of the structure of the space, the best move at any time would appear to be to subtract the largest number possible from $\mathrm{X}_{\mathrm{i}}$. However, in certain cases (where this would leave us in the ranges $[59.99,60.99]$ or $[29.99,30.99])$ the best move is actually to subtract a smaller number. Thus a straightforward hill-climbing search with a naive cost estimate, which always selects the best local move, will not find the optimum cost. 
In addition to the different move sets, we also implemented a number of different intermediate objective functions, to see if there was a relationship between objective function and move set. The cost functions are described below.

Let $\mathrm{L}$ be a partial list of length $\mathrm{N}$ with last element $\mathrm{i}$. Each cost function uses an estimate of the number of moves required to complete the list, by assuming an average decrement over all the moves. The " $\max (1000 * \mathrm{i}, 0)$ " function is to reward moves which complete the list when $i<1$, and the $1 / \mathrm{N}$ factor in function 5 encourages forward generation.

1. $f(L)=N+(i * 100)+\max (1000 * i, 1)$

("worst cost" - assumes an average decrement of 0.01 ).

2. $f(L)=N+i+\max (1000 * i, 1)$

("poor cost" - assumes an average decrement of 1 ).

3. $f(L)=N+i / 2.5+\max (1000 * i, 1)$

("average cost" - assumes an average decrement of 2.5).

4. $f(L)=N+i / 4+\max (1000 * i, 1)$

("good cost" - assumes an average decrement of 4).

5. $\mathrm{f}(\mathrm{L})=\mathrm{N}+\mathrm{i} / 5+\max (1000 * \mathrm{i}, 1)+1 / \mathrm{N}$

("best cost" - assumes an average decrement of 5).

In running these experiments, the adaptive annealing schedule of Huang et al. (1986) was used, which calculates initial temperatures, temperature decrements and equilibrium criteria by statistical sampling of the algorithm's performance. For determining the size of a backtracking move, we considered five percentage bands $(0-20 \%, 20-40 \%$, etc.). First we select a percentage band, compute the limits on backtracking that imposes, and then randomly select a backtrack height within those limits. To select the percentage band, we use the probabilistic move selection technique suggested by Hustin and Sangiovanni-Vincentelli (1987). This technique computes the average objective function change induced by each accepted move type, and updates the probability of applying a move based on these statistics. This ensures that there is a bias towards moves which are likely to have the most effect on the objective functions at each temperature. We expect large backtracks during the early stages of an annealing run, and small backtracks during the later stages. Finally, to include a degree of intelligence in the forward move selection, we have imposed a $25 \%$ probability of selecting a decrement of 5 where appropriate. 
We ran algorithms $A$ to $E$ ten times each for each of the five cost functions, and we ran $F$ and $G$ a total of ten times each (as $F$ and $G$ use the evaluated objective functions for completed designs, the different cost estimates do not apply). The results are tabulated in Table 1 . The first row of each block lists the average number of attempted moves. The second row states the number of times the algorithm converges on its best solution, as opposed to simply visiting it in the higher temperature stages, but converging on poorer local optima. The " + " symbol indicates that in a small number of cases, the algorithm converged around the best cost, but did not actually settle on it. The third row contains the average best cost over the ten runs - the numbers in brackets list the number of runs which produced a completed list if this was less than ten. The fourth row lists the best cost obtained over the ten runs, if a completed list was obtained .

\section{Discussion}

First, consider the first five algorithms (those not requiring a complete generation at each move). From these results, it can be seen that in terms of approaching the optimum, original shape annealing (A), although it does find relatively short lists, is restricted by its limited backtracking strategy. In general, B, which jumps back to higher states, produces better solutions in fewer iterations. $\mathrm{C}$ is consistently best at finding the optimum for the first four objective functions. However, it takes at least an order of magnitude more iterations than the other algorithms, and rarely converges on its best cost. The increase in the number of iterations is largely due to the irregular cost comparison and Huang et al.'s method of calculating statistics based on the average costs of the states at a given temperature. This increased number of iterations allows the algorithm to sample more of the space, and it tends to visit the optimum cost during the early to middle stages. Its inability to converge on good solutions is also caused by the irregular comparison method. The algorithm is able to backtrack at very little cost: by comparing the costs of the states at the high level, it makes its decisions without consideration of how close the previous state was to a complete list. Finally, by comparing states at that level, decisions are taken solely in terms of the local objective functions, and thus the algorithm gets caught in the "traps" at values 60 and 30. D, which uses the same backtracking move but compares the new state with the previous state, requires significantly fewer iterations, and has better convergence. E, which extends the look ahead limit, tends to converge more frequently on better solutions in less time. 
TABLE 1. Results

\begin{tabular}{llllll} 
A & 1 & 2 & 3 & 4 & 5 \\
\hline iterations & 79495 & 59545 & 54667 & 46083 & 89052 \\
converge & 0 & $0+$ & $1+$ & $7+$ & 0 \\
average & 26.9 & 27.1 & 25.9 & 21.1 & $26(2)$ \\
best & 23 & 26 & 22 & 20 & 26
\end{tabular}

\begin{tabular}{llllll} 
B & 1 & 2 & 3 & 4 & 5 \\
\hline iterations & 58811 & 51275 & 30704 & 23631 & 101590 \\
converge & $1+$ & 2 & 8 & 8 & 0 \\
average & 26.5 & 24.6 & 23.3 & 20.5 & $*$ \\
best & 24 & 21 & 22 & 20 & $*$
\end{tabular}

\begin{tabular}{llllll} 
C & 1 & 2 & 3 & 4 & 5 \\
\hline iterations & 217000 & 267858 & 330362 & 366261 & 181698 \\
converge & 0 & 0 & 0 & 0 & 0 \\
average & 20.5 & 20.4 & 20 & 20 & $21(2)$ \\
best & 20 & 20 & 20 & 20 & 21
\end{tabular}

\begin{tabular}{llllll}
$\mathrm{D}$ & 1 & 2 & 3 & 4 & 5 \\
\hline iterations & 94447 & 51503 & 49860 & 31359 & 65747 \\
converge & $1+$ & $1+$ & 4 & 10 & 0 \\
average & 24.1 & 23.5 & 22.2 & 20.6 & $21(1)$ \\
best & 23 & 21 & 21 & 20 & 21
\end{tabular}

\begin{tabular}{llllll}
$\mathrm{E}$ & 1 & 2 & 3 & 4 & 5 \\
\hline iterations & 94282 & 46986 & 48259 & 20633 & 31560 \\
converge & $0+$ & $2+$ & $4+$ & $9+$ & 8 \\
average & 23.7 & 22.9 & 21.4 & 20.4 & $20.44(9)$ \\
best & 22 & 22 & 21 & 20 & 20
\end{tabular}

F

\begin{tabular}{ll}
\hline iterations & 65662 \\
converge & 6 \\
average & 21.4 \\
best & 21 \\
& \\
G & \\
iterations & 9252 \\
converge & 8 \\
average & 20.7 \\
best & 20
\end{tabular}


Note also that the different cost estimates also play a significant role. The worst cost objective function (1) causes every forward move to be accepted, and thus all optimization is carried out during the backtracking moves. As the cost estimates improve, selection of forward moves becomes more discriminating. Generally, the solutions improve as the local cost estimates better reflect the final cost, except in the case of the "best cost" estimate (5), which generally causes the algorithms to converge in the local optima. The exception to this rule is E. It appears that the extended look ahead allows the algorithm to backtrack out of the local optima by jumping over the moves that trap the other variations, in both the "good cost" and "best cost" objective functions. For the first three cost functions, $\mathrm{E}$ is also better, but less markedly so. E's success may be attributed to the fact that it is able to jump back above local optima, and then look ahead far enough to identify better moves.

Finally, consider the performance of the two algorithms requiring complete generations. The first (F) consistently finds and converges on good solutions, in relatively few iterations, although each iteration is a longer and more complex move than in the first 5 algorithms. However, F rarely finds the optimum. $G$ finds and converges on the optimum regularly, in fewer iterations. In turn, though, each iteration for $\mathrm{G}$ is on average 10 times as complex as for $\mathrm{F}$, and the algorithm takes a correspondingly longer time to finish. The improved performance of $\mathrm{G}$ appears to be due to the sampling of final solutions compensating for the random nature of the forward generation. Suppose in the mid to late stages of a run that the move from $s_{i}$ to $s_{i+1}$ was a poor move, and that $s_{i}$ appears relatively early in the move sequence. Because we are at least in the middle of a run, we will have optimized the moves below $s_{i+1}$ to some extent. Suppose also that we now backtrack to $s_{i}$, move forward to $s_{i+1}{ }^{\prime}$ and then complete the generation, and that the move to $s_{i+1}$ is better than the move to $s_{i+1}$ in global terms. Because the list completion from $s_{i+1}$ is random, it is unlikely to result in a better final list than the part-optimized completion from $\mathrm{s}_{\mathrm{i}+1}$, and thus it is unlikely to be accepted, even though the move from $s_{i}$ to $s_{i+1}$ ' was better. For this problem, the sampling of completions in $\mathrm{G}$ appears to be sufficient to direct the algorithm towards the best moves.

Although E appears to be the most successful, care should be taken in assuming that it is the best algorithm to use. The particular look ahead limit imposed here may be suited to the particular local optima found in the design space. In addition, it is probably also significant that the design space is relatively uniform away from these optima, and a simple, regular cost estimate is a good characterization of the space. With irregular spaces, it is not clear that E would perform so well. In such cases, G, although slower, 
may prove superior. More research is required relating the characteristics of the design space to the move set and intermediate objective functions.

\section{Conclusions}

Shape annealing is a robust, efficient method for generating good solutions in large design spaces. Its performance for those applications where a forward move restricts the design space can be improved by modifying its backtracking moves, incorporating a jump backtrack and limited look ahead. Annealing based on the sampling of completed designs also improves performance, although at the expense of time.

\section{Acknowledgments}

The authors would like to thank the National Science Foundation under grants DDM-9300196 and DDM-925-8090, and United Technologies for supporting this work. We also thank the members of the Computational Design Laboratory, Carnegie Mellon University, for their contributions to this work; in particular, Simon Szykman provided his implementation of the dynamic simulated annealing algorithm, and spent a lot of time helping us understand its behavior.

\section{References}

Brown, K. N., Sims Williams, J. H. and McMahon, C. A.: 1992, Grammars of features in design, in J. S. Gero (ed.) Artificial Intelligence in Design '92, Kluwer Academic Publishers, Dordrecht, pp. 287-306.

Brown, K. N., McMahon, C. A. and Sims Williams, J. H.: 1993, The role of formal grammars in the engineering design process, Internal Report, Department of Engineering Mathematics, University of Bristol.

Brown, K. N., McMahon, C. A. and Sims Williams, J. H.: 1994a, Features, aka The Semantics of a Formal Language of Manufacturing, Research in Engineering Design (to appear).

Brown, K. N., McMahon, C. A. and Sims Williams, J. H.: 1994b, Describing process plans as the formal semantics of a language of shape, Artificial Intelligence in Engineering (to appear).

Cagan, J., and Mitchell, W. J.: 1993, Optimally directed shape generation by shape annealing, Environment and Planning B, 20, 5-12.

Cagan, J.: 1994a, A shape annealing solution to the constrained geometric knapsack problem, Computer-Aided Design, 28(10), 763-769.

Cagan, J.: 1994b, Research issues in the application of design grammars, in J. S. Gero and E. Tyugu (eds), Formal Design Methods for CAD, North-Holland, Amsterdam, pp. 191198.

Cagan, J. and Kotovsky, K.: 1994, The generation of the objective function in a simulated annealing model of problem solving, Working Paper, available from the authors.

Carlson, C.: 1994a, A tutorial introduction to grammatical programming, in J. S. Gero and E. Tyugu (eds), Formal Design Methods for CAD, North-Holland, Amsterdam, pp. 73-84. 
Carlson, C.: 1994b, Design space description formalisms, in J. S. Gero and E. Tyugu (eds), Formal Design Methods for CAD, North-Holland, Amsterdam, pp. 121-131.

Fitzhorn, P. A.: 1989, A computational theory of design, Preprints NSF Engineering Design Research Conference, College of Engineering, University of Massachusetts, Amherst.

Flemming, U.: 1987, More than the sum of their parts: The grammar of Queen Anne houses, Environment and Planning $B, 14,323-350$.

Heisserman, J. and Woodbury, R.: 1994, Geometric design with boundary solid grammars, in J. S. Gero and E. Tyugu (eds), Formal Design Methods for CAD, North-Holland, Amsterdam, pp. 85-105.

Huang, M. D., Romeo, R. and Sangiovanni-Vincentelli, A.: 1986, An efficient general cooling schedule for simulated annealing algorithm, ICCAD-86 IEEE International Conference on CAD, Digest of Technical Papers, Santa Clara, CA, pp. 381-384.

Hustin, S. and Sangiovanni-Vincentelli, A.: 1987, TIM, a new standard cell placement program based on the simulated annealing algorithm, IEEE Physical Design Workshop on Placement and Floorplanning, Hilton Head, SC.

Kirkpatrick, S., Gelatt, C. D. Jr. and Vecchi, M. P.: 1983, Optimization by simulated annealing, Science, 220(4598), 671-679.

Knuth, D.: 1968, Semantics of context-free languages, Mathematical Systems Theory, 2(2), 127-145.

McMahon, C. A., Sims Williams, J. H. and Brown, K. N.: 1993, A transformation model for the integration of design computing, International Conference on Engineering Design (ICED'93), The Hague.

Penjam, J.: 1990, Computational and attribute models of formal languages, Theoretical Computer Science, 71, 241-264.

Reddy, G. and Cagan, J.: 1995, An improved shape annealing algorithm for truss topology generation, ASME Journal of Mechanical Design, 117(2A), 315-321.

Rinderle, J. R.: 1991, Grammatical approaches to engineering design, Part II: Melding configuration and parametric design using attribute grammars, Research in Engineering Design, 2(3), 137-146.

Schmidt, L. C. and Cagan, J.: 1995, Recursive annealing: A computational model for machine design, Research in Engineering Design, 7, 102-125.

Stiny, G. and Mitchell, W. J.: 1978, The Palladian Grammar, Environment and Planning B, $5,5-18$.

Stiny, G.: 1980, Introduction to shape and shape grammars, Environment and Planning B, 7, 343-351.

Stiny, G. and March, L.: 1981, Design machines, Environment and Planning B, 8, 245-255.

Stiny, G.: 1981, A note on the description of designs, Environment and Planning B, 8, 257267.

Stiny, G.: 1991, The algebras of design, Research in Engineering Design, 2(3), 171-181.

Szykman, S. and Cagan, J.: 1995, A simulated annealing approach to three dimensional component packing, ASME Journal of Mechanical Design, 117(2A), 308-314. 


\section{Appendix}

Symbols: $\{<,>\}$

Initial list: $<100>$

$\mathrm{X}$ and $\mathrm{Y}$ are real-valued variables.

Rules:

1) $\mathrm{X}>\rightarrow \mathrm{X} \max (\mathrm{X}-\mathrm{Y}, 0)>$ where $\mathrm{X}<29.99$ or $32 \leq \mathrm{X}<59.99$ or $62 \leq \mathrm{X} \leq 100$ and $\mathrm{Y} \in\{0.01,0.02, \ldots, 4.99,5\}$

2) $X>\rightarrow X 55>\quad$ where $61 \leq X<62$

3) $X>\rightarrow$ X $59.99>\quad$ where $60 \leq X<61$

4) $59.99>\rightarrow 59.9959 .98>$

5) $\mathrm{X}>\rightarrow \mathrm{X} 25>\quad$ where $31 \leq \mathrm{X}<32$

6) $\mathrm{X}>\rightarrow \mathrm{X} 29.99>\quad$ where $30 \leq \mathrm{X}<31$

7) $29.99>\rightarrow 29.9929 .98>$ 